Impfpflicht

\section{In einer liberalen Gesellschaft}

Der Deutsche Bundestag wird bei der Frage nach einer allgemeinen Impfpflicht vor einer schwierigen Entscheidung stehen. Gegner und Skeptiker einer allgemeinen Impfpflicht sehen einen Widerspruch zu den Grundsätzen einer liberalen Ordnung, schließlich ist eine Impfung ein Eingriff in den eigenen Körper. Soll das kollektive Interesse über dem individuellen Persönlichkeitsrecht stehen? Kann in diesem Konflikt die Wirtschaftswissenschaft einen Beitrag leisten? Ja, man muss allerdings mehr als 50 Jahre zurückgehen, um auf das in den 1970er und 1980er Jahren intensiv diskutierte Theorem von der Unmöglichkeit eines Paretianischen Liberalismus zu stoßen. Es ist eines der vielen fundamentalen Theoreme eines der wichtigsten Denker unserer Zeit, Amartya Sen (1970). Als Personen sind wir immer wieder kollektiven Entscheidungen unterworfen: vom Straßenverkehrsrecht bis zum Steuerrecht. Sie engen unseren Handlungs- und Entscheidungsspielraum ein. In einer liberalen Gesellschaft müssen aber auch individuelle Entscheidungen immer möglich sein, besonders wenn es die eigene, private Sphäre betrifft. Ob wir auf dem Rücken oder auf der Schulter schlafen: das ist keine Entscheidung des Staates. Dann, so könnte man argumentieren, sollte die Entscheidung für oder gegen eine Impfung auch eine Entscheidung der privaten Sphäre sein. Sen suchte nach einer allgemeingültigen Entscheidungsregel, die zumindest diesen liberalen Gedanken erfüllt. Genau genommen bezeichnet der "minimale Liberalismus“ die Möglichkeit von mindestens zwei Personen über jeweils ein Tupel aus der Menge möglicher Alternativen gesellschaftlich ausschlaggebend zu sein. Darüber hinaus soll diese Entscheidungsregel paretianisch sein, wenn sich also beide Individuen darin einig sind, dass Alternative $x$ der Alternative y vorzuziehen ist, dann sollte y nicht die kollektive Entscheidung sein. Allgemeingültig heißt, dass alle denkbaren Präferenzen diese beiden Eigenschaften erfüllen müssen.

Betrachten wir nun zwei Personen, eine Impfbefürworterin (B) und einen Impfskeptiker (S). Beide haben Präferenzen über das folgende Tripel an Alternativen: $x$ : beide werden geimpft, $y$ : nur B wird geimpft und $z$ : nur $S$ wird geimpft. Die Präferenzordnung der Impfbefürworterin sei $x$ vor $y$ und $y$ vor $z$ : am liebsten ist ihr, alle sind geimpft, aber wenn es nicht möglich ist, dann will sie lieber geimpft sein als der Impfskeptiker. Dieser lehnt die allgemeine Impfpflicht ( $\mathrm{x}$ ) völlig ab und präferiert an erster Stelle,

(C) Der/die Autor:in 2021. Open Access: Dieser Artikel wird unter der Creative Commons Namensnennung 4.0 International Lizenz veröffentlicht (creativecommons.org/licenses/by/4.0/deed.de).

Open Access wird durch die ZBW - Leibniz-Informationszentrum Wirtschaft gefördert. dass sich nur B impft. Seine Präferenzordnung lautet in absteigender Präferenz also y, z, x. Minimaler Liberalismus bedeute nun, dass $B$ die gesellschaftliche Entscheidung $\mathrm{x}$ vor $\mathrm{y}$ determinieren kann. $\mathrm{S}$ determiniert $\mathrm{z}$ vor $\mathrm{x}$. Aus dem Pareto-Prinzip folgt zudem y vor $z$. Das Ergebnis ist ein klassischer Zyklus. Die Alternative $z$ steht gesellschaftlich vor $\mathrm{x}, \mathrm{x}$ wiederum steht gesellschaftlich vor $\mathrm{y}$, und die Alternative y vor $z$. Der Mangel an Transitivität ermöglicht also keine Entscheidung. Wenn es aber nicht möglich ist, diese elementaren Bedingungen zu erfüllen, kann es auch keine komplexere Entscheidungsregel geben. Natürlich provozierte Sen mit der Veröffentlichung eine rege Diskussion, schließlich hinterfragte er fundamentale Glaubenssätze liberaler Wirtschaftsordnungen. Aus der Chicago-Schule wurde propagiert, dass eine Lösung in einem Vertrag gefunden werden kann. Angewendet auf das Beispiel würde $B$ auf ihr Recht verzichten, $x$ vor y zu determinieren (Gibbard, 1974). Dann folgt aus der liberalen Entscheidung von $S(z$ vor $x$ ) und dem ParetoPrinzip ( $y$ vor $z$ ) die gesellschaftliche Ordnung y vor $z$ und $z$ vor $x$. Die Impfbefürworterin würde durch ihren Verzicht ihre liebste Präferenz durchsetzen, die es jenseits der allgemeinen Impfpflicht gibt.

Nun sind Pareto-superiore Verträge in der Ökonomie recht beliebt, aber tragen sie wirklich zur Lösung bei? Sen antwortete darauf mit dem Hinweis auf die „Paretianische Epidemie“ (Sen, 1976): hat nur eine Person ein liberales Recht, so kann sie (hier: der Impfskeptiker) die gesamte gesellschaftliche Präferenzordnung bestimmen: $y, z, x$. Seine Präferenzordnung drückt er vollständig der Gesellschaft auf. Das Pareto-Prinzip führt zu dem, was in der Social-Choice-Literatur als diktatorische Eigenschaft bezeichnet wird. Das kann nicht das Ziel einer liberalen Gesellschaftsordnung sein. Soweit der Bundestag also zwischen individuellen Grundrechten und kollektiver Rationalität zu entscheiden hat, gibt es keine allgemeine Entscheidungsregel. Will man den liberalen Grundgedanken der Verträge als Lösung dennoch nicht abschreiben, so wäre eine allgemeine Impfpflicht, von der man sich durch einen vertraglichen Verzicht auf intensivärztliche Behandlung befreien kann, denkbar. Inwieweit das ethisch und rechtlich möglich ist, dazu wiederum kann die ökonomische Theorie tatsächlich wenig beitragen.

Salvatore Barbaro Johannes Gutenberg-Universität Mainz sbarbaro@uni-mainz.de

\section{Literatur}

Gibbard, A. (1974), Pareto-consistent libertarian claim, JET.

Sen, A. (1970), The Impossibility of a Paretian Literal, J Polit Econ Sen, A. (1976), Liberty, Unanimity and Rights, Economica. 